Rev. Int. Contam. Ambie. 34 (3) 373-382, 2018

DOI: 10.20937/RICA.2018.34.03.01

\title{
CARACTERIZACIÓN QUÍMICA Y FÍSICA DEL BAGAZO DE AGAVE TEQUILERO COMPOSTADO CON BIOSÓLIDOS DE VINAZA COMO COMPONENTE DE SUSTRATOS PARA CULTIVOS EN CONTENEDOR
}

\author{
Marcos Rafael CRESPO GONZÁLEZ ${ }^{1}$, Diego Raymundo GONZÁLEZ EGUIARTE ${ }^{1}$, \\ Ramón RODRÍGUEZ MACÍAS ${ }^{1 *}$, José Ariel RUIZ CORRAL ${ }^{2}$ y Noé DURÁN PUGA ${ }^{2}$
}

\footnotetext{
${ }^{1}$ Universidad de Guadalajara. Carretera a Nogales km. 15.5, Predio Las Agujas, Apartado Postal 52-93, Zapopan, Jalisco, México, C. P. 45020

2 Instituto Nacional de Investigaciones Forestales Agrícolas y Pecuarias. Kilómetro 8, Carretera Libre TepatitlánLagos de Moreno, Tepatitlán de Morelos, Jalisco, México, C. P. 47600

*Autor para correspondencia: ramonrod@cucba.udg.mx
}

(Recibido junio 2016; aceptado octubre 2017)

Palabras clave: Agave tequilana Weber, residuos orgánicos, compostaje, cultivos protegidos

\section{RESUMEN}

El presente estudio tuvo como objetivo caracterizar las propiedades químicas y físicas del bagazo de agave tequilero compostado con biosólidos de vinaza en formulaciones con polvo de coco, para compararlas con sustratos comerciales utilizados para cultivos en contenedor, con el fin de valorar el potencial de aprovechamiento de los dos desechos más importantes de la industria tequilera. Los sustratos evaluados fueron polvo de coco, turba canadiense, mezclas de polvo de coco con tres niveles de compost: $30 \%$, $50 \%$ y $70 \%(\mathrm{v} / \mathrm{v})$, y compost $100 \%$. Los factores químicos a analizar fueron: materia orgánica, $\mathrm{pH}$, conductividad eléctrica, capacidad de intercambio catiónico, carbono orgánico total, relación $\mathrm{C} / \mathrm{N}$, nitrógeno total, fósforo, potasio y magnesio; las físicas fueron: densidad aparente, densidad real, espacio poroso total, agua fácilmente disponible, agua de reserva, agua total disponible y distribución del tamaño de partículas. Se pudo demostrar que las formulaciones de compost de bagazo de agave con polvo de coco podrían reemplazar el uso de polvo de coco y turba, ya que poseen características químicas y físicas satisfactorias para ser utilizadas como sustratos para plantas cultivadas en contenedor. Los tratamientos mejor evaluados fueron compost $30 \%, 50 \%$ y $100 \%$. El aprovechamiento de los desechos compostados podría traer consigo otros beneficios, tales como la reducción de la contaminación ambiental a través de la producción local de sustratos destinados al sector de los cultivos protegidos, el cual tiene una demanda cada vez más creciente de estos materiales.

Key words: Agave tequilana Weber, organic residues, compost, protected agriculture

\begin{abstract}
The objectives of this study were to determine the chemical and physical properties of tequila blue agave bagasse composted with tequila vinasses' biosolids in coconut based powder substrates for potted plants and to compare their performance against two commercial substrates. The substrates evaluated included coconut powder,
\end{abstract}


Canadian peat, $100 \%$ compost, and 3-coconut powder based mixtures containing $30 \%$, $50 \%$, and $70 \%$ compost. Chemical parameters analyzed comprise organic matter, $\mathrm{pH}$, conductivity, cation exchange capacity, total organic carbon, $\mathrm{C} / \mathrm{N}$ ratio, total nitrogen, phosphorus, potassium, and magnesium. The physical properties evaluated included bulk density, particle density, total porous spaces, free water, bound water, absorbed water and particle size distribution. We were able to demonstrate that the formulations of agave bagasse compost and coconut powder could replace coconut powder and peat, since their physical and chemical characteristics are suitable for its use as substrate for plants cultivated in containers. The treatments with better results were those with 30 $\%, 50 \%$, and $100 \%$ compost. Composting waste materials provide additional benefits including a reduction in environmental pollution as well as the local production of substrates for protected agriculture systems, which require substantial quantities of these type of materials.

\section{INTRODUCCIÓN}

La cadena productiva del agave al tequila es muy importante en el sector agroindustrial de México ya que miles de familias que dependen económicamente de ella. Sin embargo, este hecho tiene un costo ambiental, ya que la producción de tequila genera también grandes cantidades de residuos. Por ejemplo, para producir un litro de tequila se desechan aproximadamente entre 10 y $12 \mathrm{~L}$ de vinazas y de 3.5 a $7 \mathrm{~kg}$ de bagazo de agave (CNIT 2013). Si se considera que anualmente se producen más de 228 millones de litros de tequila (CRT 2016), es fácil deducir que existe una considerable cantidad de residuos que representan un gran desafío para manejarlos adecuadamente y evitar riesgos de contaminación ambiental.

Además del bagazo de agave, algunas empresas tequileras generan biosólidos, que son el producto del procesamiento de aguas de desecho (vinazas) a través de plantas de tratamiento de aguas residuales (PTAR). Esta es una tecnología que permite no sólo dar tratamiento a las vinazas, sino también reducir los contaminantes presentes a niveles permisibles para su manejo y aprovechamiento. Los biosólidos llegan a tener una concentración de agua que varía entre 94 y $97 \%$, y pueden ser útiles como fertilizantes porque contienen varios nutrientes tales como nitrógeno $(6.9 \%)$, fósforo $(1.88 \%)$ y potasio $(1.32 \%)$ principalmente (EPA 2000).

Aunque existen varias alternativas de aprovechamiento de los residuos sólidos y líquidos de la industria tequilera, el compostaje aeróbico ha resultado ser hasta ahora la técnica más utilizada ya que puede reciclarse el bagazo junto con las vinazas o los biosólidos; el compost así producido puede emplearse como enmienda para suelos agrícolas. A este respecto, algunos trabajos consignan que el compost humectado únicamente con agua también puede utilizarse como sustrato para la producción de plantas (Rodríguez et al. 2010, Crespo et al. 2013). Sin embargo, no existen reportes de compost elaborado con biosólidos y usado como sustrato para el cultivo de plantas en contenedor.

Por otra parte, la turba o "peat moss" ${ }^{\circledR}$ es un sustrato orgánico de uso común en el mundo, pero en México es importado a un alto costo, aunque en los países de origen existen restricciones para su explotación, ya que daña el ecosistema de humedales. Por su parte, el polvo de coco es también un sustrato muy utilizado en México, ya que se produce y comercializa a un menor costo que la turba, sin embargo, aun así no es un sustrato económico por lo que es muy importante investigar los materiales locales que puedan reemplazar a los comerciales antes citados.

Con base en lo anterior, el objetivo de la presente investigación fue caracterizar las propiedades químicas y físicas del bagazo de agave tequilero compostado con biosólidos de vinaza en formulaciones con polvo de coco, para compararlas con sustratos comerciales para cultivos en contenedor, con el fin de valorar el potencial de aprovechamiento de los dos desechos más importantes de la industria tequilera.

\section{MATERIALES Y MÉTODOS}

El estudio se llevó a cabo en el rancho El Indio, propiedad de la empresa Tequila Sauza, S.A, localizada en el municipio de Tequila, Jalisco, cuyas coordenadas son $20^{\circ} 53^{\prime} 41^{\prime \prime} \mathrm{N}$ y $103^{\circ} 50^{\prime} 10.50^{\prime}$ "W. Se utilizó compost de bagazo de agave producido por la empresa mediante el sistema de pilas aireadas mezcladas con biosólidos de vinazas provenientes de la planta tratadora de aguas residuales (PTAR) localizada en el mismo lugar. En el cuadro I, se muestra la composición química del bagazo de agave 
CUADRO I. CARACTERÍSTICAS FÍSICAS Y QUÍMICAS DEL BAGAZO DE AGAVE Y BIOSÓLIDOS (Rodríguez et al. 2013).

\begin{tabular}{lcc}
\hline Análisis & Bagazo de agave & Biosólidos \\
\hline $\mathrm{pH}^{*}$ & 4.8 & 8.51 \\
$\mathrm{CE}(\mathrm{mS} / \mathrm{cm})^{*}$ & 0.14 & 4.12 \\
$\mathrm{MO}(\%)$ & 88.60 & 78.00 \\
$\mathrm{Ceniza}(\%)$ & 11.40 & 22.00 \\
$\mathrm{COT}(\%)$ & 51.51 & 45.37 \\
Relación C/N & 66.90 & 6.61 \\
$\mathrm{NTK}(\%)$ & 0.77 & 6.87 \\
$\mathrm{P}(\%)$ & 0.07 & 1.88 \\
$\mathrm{~K}(\%)$ & 0.26 & 1.32 \\
$\mathrm{Na}(\%)$ & 0.05 & 1.92 \\
$\mathrm{Ca}(\%)$ & 3.68 & 1.98 \\
$\mathrm{Mg}(\%)$ & 0.23 & 0.59 \\
$\mathrm{Cu}(\mathrm{ppm})$ & 4.85 & 143.95 \\
$\mathrm{Fe}(\mathrm{ppm})$ & 371.00 & 6010.10 \\
$\mathrm{Zn}(\mathrm{ppm})$ & 30.49 & 2219.58 \\
$\mathrm{~B}(\mathrm{ppm})$ & 161.23 & 264.56 \\
$\mathrm{Ar}(\mathrm{ppm})$ & nd & 3.45 \\
$\mathrm{Cd}(\mathrm{ppm})$ & nd & 31.0 \\
$\mathrm{Cu}(\mathrm{ppm})$ & nd & 106 \\
$\mathrm{Cr}(\mathrm{ppm})$ & nd & 40.1 \\
$\mathrm{Hg}(\mathrm{ppm})$ & nd & $<0.500$ \\
$\mathrm{Ni}(\mathrm{ppm})$ & nd & 38.8 \\
$\mathrm{~Pb}(\mathrm{ppm})$ & nd & 632 \\
\hline
\end{tabular}

* base húmeda. $\mathrm{CE}=$ conductividad eléctrica, $\mathrm{MO}=$ materia orgánica base seca, $\mathrm{COT}=$ carbono orgánico total, $\mathrm{NTK}=$ nitrógeno total Kjeldahl, nd = no determinado

sin compostar, así como la de los biosólidos (Rodríguez et al. 2013).

Con el compost se formularon cuatro sustratos con las siguientes formulaciones: 1) $30 \%$ de compost con polvo de coco $(\mathrm{v} / \mathrm{v}), 2) 50 \%$ de compost con polvo de coco $(\mathrm{v} / \mathrm{v}), 3) 70 \%$ de compost con polvo de coco (v/v) y 4) compost al $100 \%$. Estos materiales se compararon con dos sustratos comerciales: polvo de coco producido en Michoacán, México y turba del musgo Sphagnum de Canadá.

\section{Análisis químicos}

El contenido de nitrógeno total (NTK) fue determinado por el procedimiento semi micro Kjeldahl (TMECC 2001). Las cenizas por combustión en una mufla a $550^{\circ} \mathrm{C}$ (TMECC 2001). La materia orgánica (MO) por cálculo del peso de cenizas menos el peso seco de la muestra original. El carbono orgánico total (COT) por cálculo (MO/1.724) (Gouleke 1977). La relación $\mathrm{C} / \mathrm{N}$ por cálculo con base a los análisis anteriores de carbono y nitrógeno total. La conductividad eléctrica (CE) y el pH de los materiales fueron determinados en extractos de agua destilada en una relación peso/volumen de 1:5 (TMECC 2001), utilizando equipo marca Hanna ${ }^{\circledR}$ HI 4321 y HI 2210 respectivamente. La capacidad de intercambio catiónico se determinó en acetato de amonio $1 \mathrm{~N} \mathrm{y}$ $\mathrm{pH}$ 7. El fósforo se midió con un espectrofotómetro marca Spectro ${ }^{\circledR}$ 23RS (TMECC 2001). El potasio, el calcio y el magnesio fueron determinados con un espectrofotómetro de absorción atómica marca Varian ${ }^{\circledR}$ AA240FS (TMECC 2001).

\section{Análisis físicos}

Las determinaciones se basaron en las técnicas recomendadas por Ansorena (1994), las cuales fueron: densidad aparente (Da), densidad real (Dr), espacio poroso total (EPT), distribución del tamaño de partículas mediante el uso de cribas: 9.52, 4.70, $2.00,1.00,0.42,0.25$ y $0.15 \mathrm{~mm}$ colocadas en un agitador electromagnético Ro-Tap ${ }^{\circledR}$ modelo Rx-29 de vaivén (Martínez 1993). Finalmente, se determinaron las curvas de liberación de agua por el método de la columna colgante (De Boodt y Verdonck 1974), y a partir de dicha medición, se obtuvieron los valores de agua fácilmente disponible (AFD), agua de reserva (AR) agua total disponible (ATD) y porosidad de aire (PA).

\section{Evaluación estadística de resultados}

Se aplicó un diseño completamente al azar con seis tratamientos y tres repeticiones. Con los resultados se efectuó un análisis de varianza y la prueba de comparación de medias de Tukey con el paquete estadístico Minitab 17 (2015).

\section{RESULTADOS Y DISCUSIÓN}

\section{Propiedades químicas de los sustratos}

En el cuadro II se presentan las características químicas del polvo de coco, turba y los sustratos a base de compost formulados a 30, 50 y $70 \%(\mathrm{v} / \mathrm{v})$, mezclados con polvo de coco, y compost $100 \%$, con los resultados de la prueba de Tukey; en tanto, el cuadro III muestra el resumen del análisis de varianza obtenido de las características químicas de los sustratos evaluados.

Materia orgánica (MO). En el cuadro II, se observa que el polvo de coco y la turba mostraron un contenido de MO típico de materiales con bajo grado de mineralización (Day y Shaw 2001) y se ubicaron dentro del parámetro sugerido para sustratos (> $80 \%$ ). Los tratamientos con compost mostraron valores claramente más bajos con un intervalo entre $37.83 \%$ y $48.2 \%$, lo cual es resultado del proceso 
CUADRO II. CARACTERÍSTICAS QUÍMICAS DE LOS SUSTRATOS COMERCIALES Y MEZCLAS A BASE DE COMPOST

\begin{tabular}{|c|c|c|c|c|c|c|c|}
\hline \multirow{2}{*}{ Análisis } & \multirow{2}{*}{$\begin{array}{l}\text { Polvo de } \\
\text { coco }\end{array}$} & \multirow{2}{*}{ Turba } & \multicolumn{4}{|c|}{ Compost \% (v/v) } & \multirow{2}{*}{ Recomendados $\dagger$} \\
\hline & & & 30 & 50 & 70 & 100 & \\
\hline MO (\%) & $91.80^{\mathrm{b}}$ & $94.50^{\mathrm{a}}$ & $48.20^{\mathrm{c}}$ & $45.20^{\mathrm{d}}$ & $37.80^{\mathrm{e}}$ & $38.70^{\mathrm{e}}$ & $>80$ \\
\hline $\mathrm{pH}$ (en agua $1: 5)$ & $5.70^{\mathrm{c}}$ & $4.40^{\mathrm{d}}$ & $6.60^{\mathrm{b}}$ & $7.10^{\mathrm{b}}$ & $7.30^{\mathrm{b}}$ & $8.50^{\mathrm{a}}$ & $5.2-6.3$ \\
\hline CIC meq $100^{-1}$ & $138.80^{\mathrm{b}}$ & $162.10^{\mathrm{a}}$ & $84.50^{\mathrm{de}}$ & $110.50^{\mathrm{c}}$ & $97.50^{\mathrm{cd}}$ & $74.50^{\mathrm{e}}$ & $>20$ \\
\hline $\mathrm{CE}\left(\mathrm{dS} \mathrm{m} \mathrm{m}^{-1}\right)$ & $0.31^{\mathrm{c}}$ & $0.24^{\mathrm{c}}$ & $0.80^{\mathrm{b}}$ & $1.40^{\mathrm{a}}$ & $1.50^{\mathrm{a}}$ & $0.43^{\mathrm{c}}$ & $0.75-3.49$ \\
\hline $\operatorname{COT}(\%)$ & $53.25^{\mathrm{a}}$ & $54.79^{\mathrm{a}}$ & $27.90^{\mathrm{b}}$ & $26.20^{\mathrm{b}}$ & $21.90^{\mathrm{c}}$ & $22.80^{\mathrm{c}}$ & $\mathrm{SD}$ \\
\hline $\mathrm{C} / \mathrm{N}$ & $111.47^{\mathrm{a}}$ & $71.16^{\mathrm{b}}$ & $30.70^{\mathrm{c}}$ & $25.70^{\mathrm{d}}$ & $19.90^{\mathrm{e}}$ & $16.30^{\mathrm{e}}$ & $20-40$ \\
\hline NTK $(\%)$ & $0.48^{\mathrm{d}}$ & $0.77^{\mathrm{c}}$ & $0.91^{\mathrm{bc}}$ & $1.02^{\mathrm{bc}}$ & $1.10^{\mathrm{ab}}$ & $1.38^{\mathrm{a}}$ & $0.01-0.012$ \\
\hline $\mathrm{P}(\%)$ & $0.09^{c}$ & $0.03^{\mathrm{c}}$ & $0.18^{\mathrm{b}}$ & $0.21^{\mathrm{ab}}$ & $0.24^{\mathrm{ab}}$ & $0.28^{\mathrm{a}}$ & $0.0006-0.001$ \\
\hline $\mathrm{K}(\%)$ & $1.63^{\mathrm{a}}$ & $0.07^{\mathrm{c}}$ & $0.34^{\mathrm{b}}$ & $0.37^{\mathrm{b}}$ & $0.32^{\mathrm{b}}$ & $0.38^{\mathrm{b}}$ & $0.015-0.025$ \\
\hline $\mathrm{Ca}(\%)$ & $0.26^{\mathrm{c}}$ & $0.66^{\mathrm{c}}$ & $3.60^{\mathrm{b}}$ & $4.10^{\mathrm{b}}$ & $4.87^{\mathrm{ab}}$ & $5.70^{\mathrm{a}}$ & $>0.02$ \\
\hline $\operatorname{Mg}(\%)$ & $0.11^{\mathrm{c}}$ & $0.38^{\mathrm{b}}$ & $0.27^{\mathrm{bc}}$ & $0.31^{\mathrm{b}}$ & $0.35^{\mathrm{b}}$ & $0.58^{\mathrm{a}}$ & $>0.007$ \\
\hline
\end{tabular}

$\mathrm{MO}=$ materia orgánica base seca, $\mathrm{CIC}=$ capacidad de intercambio catiónico, $\mathrm{CE}=$ conductividad eléctrica, $\mathrm{COT}=$ carbono orgánico total, $\mathrm{C} / \mathrm{N}=$ relación carbono/nitrógeno, $\mathrm{NTK}=$ nitrógeno total Kjeldahl, $\mathrm{SD}=\sin$ datos, $\dagger$ Ansorena (1994) y Noguera et al. (2003). Medias con la misma letra en cada columna son estadísticamente iguales $(\mathrm{p}<0.001)$

CUADRO III. RESUMEN DEL ANÁLISIS DE VARIANZA DE LOS ANÁLISIS QUÍMICOS DE LOS SUSTRATOS

\begin{tabular}{lccc}
\hline Análisis & $\begin{array}{c}\text { Error } \\
\text { experimental } \\
(\mathrm{s})\end{array}$ & $\begin{array}{c}\text { Coeficiente de } \\
\text { determinación } \\
\text { ajustado }\left(\mathrm{R}^{2}\right)(\%)\end{array}$ & $\begin{array}{c}\text { Nivel de } \\
\text { significancia } \\
(\mathrm{p})\end{array}$ \\
\hline $\mathrm{MO}(\%)$ & 0.7688 & 99.90 & 0.000 \\
$\mathrm{pH}$ & 0.3488 & 93.18 & 0.000 \\
$\mathrm{CIC}$ & 6.8740 & 95.45 & 0.000 \\
$\mathrm{CE}$ & 0.0663 & 98.39 & 0.000 \\
$\mathrm{COT}$ & 1.0460 & 99.48 & 0.000 \\
$\mathrm{C} / \mathrm{N}$ & 1.6700 & 99.78 & 0.000 \\
$\mathrm{NTK}$ & 1.1025 & 88.05 & 0.000 \\
$\mathrm{P}$ & 0.0302 & 90.09 & 0.000 \\
$\mathrm{~K}$ & 0.0354 & 99.54 & 0.000 \\
$\mathrm{Ca}$ & 0.5308 & 93.84 & 0.000 \\
$\mathrm{Mg}$ & 0.0526 & 85.81 & 0.000 \\
\hline
\end{tabular}

$\mathrm{MO}=$ materia orgánica base seca, $\mathrm{CIC}=$ capacidad de intercambio catiónico, $\mathrm{CE}=$ conductividad eléctrica, $\mathrm{COT}=$ carbono orgánico total, $\mathrm{C} / \mathrm{N}=$ relación carbono/nitrógeno, $\mathrm{NTK}=$ nitrógeno total Kjeldahl

de mineralización a que se someten los materiales orgánicos durante el compostaje (Day y Shaw 2001). El resultado con el compost al $100 \%(38.7 \%)$ fue un poco mayor al reportado por Íñiguez et al. (2005) para compost de bagazo de agave que fue de $31.4 \%$; no obstante, estos resultados coinciden con $\mathrm{He}$ et al. (1995), quienes señalaron que la MO de los compost en general varía entre 23.2 y $85.7 \%$, dependiendo del tipo de materia prima utilizada. Se detectaron diferencias estadísticas altamente significativas $(p<0.001)$ entre los tratamientos debido al material orgánico utilizado (Pire y Pereira 2003).

Potencial de hidrógeno (pH). Solamente el polvo de coco registró un $\mathrm{pH}$ dentro de los límites recomendados y se ubicó dentro del intervalo reportado por Maher et al. (2008) para este material, es decir, entre 4.8 y 6.9. La turba presentó el $\mathrm{pH}$ más ácido (4.4), lo cual es típico de las turbas canadienses (Alexander 2001). Por otra parte, las mezclas de compost mostraron valores mayores de $\mathrm{pH}$, mismos que variaron entre 6.60 y 7.30. En tanto que el compost al $100 \%$ dio por resultado un $\mathrm{pH}$ de 8.5 , el cual fue mayor al reportado por Íñiguez y Crohn (2004) y Rodríguez et al. (2010), quienes reportaron un $\mathrm{pH}$ de 7.2 y 7.4, respectivamente, para compost de bagazo de agave tequilero. El alto valor del $\mathrm{pH}$ de los compost tiene dos orígenes. Por un lado, el desprendimiento de amoníaco proveniente de la descomposición de las proteínas (Soliva 1998) y por el otro, la generación de carbonato de calcio como resultado de la combinación del carbonato (producto de la reacción $\mathrm{CO}_{2}$ $+\mathrm{H}_{2} \mathrm{O}$ ) con el calcio liberado del material vegetal en proceso de descomposición (Bucher 2008). Las diferencias estadísticas entre tratamientos fueron altamente significativas $(\mathrm{p}<0.001)$.

Capacidad de intercambio catiónico (CIC). Todos los tratamientos difirieron estadísticamente $(\mathrm{p}<$ 0.001) y de acuerdo con Noguera et al. (2003), todos los materiales evaluados cumplen la recomendación para sustratos (>20 meq/100 g). El valor de CIC para 
el polvo de coco $(138.8 \mathrm{meq} / 100 \mathrm{~g})$ fue mayor al registrado por Abad et al. (2002) (95.4 meq/100 g), mientras que para la turba, Burés (1997), reporta $115 \mathrm{meq} / 100 \mathrm{~g}$, valor menor al de la turba del presente trabajo $(162.1 \mathrm{meq} / 100 \mathrm{~g})$. En tanto, los sustratos con mezclas de compost variaron entre 84.5 y 110.5 meq/100 g, mientras que el compost al $100 \%$ dio por resultado $74.5 \mathrm{meq} / 100 \mathrm{~g}$, que fue superior a los $41.0 \mathrm{meq} / 100 \mathrm{~g}$ reportado por Rodríguez et al. (2010) para compost de bagazo de agave.

Cabe hacer mención que Harada et al. (1981) han sugerido que un valor de CIC $>60 \mathrm{meq} / 100$ g podría ser un indicador de madurez de compost. Bajo este criterio, el compost de bagazo utilizada en la presente investigación cubre esta recomendación.

Conductividad eléctrica (CE). La CE del polvo de coco y la turba fueron menores al óptimo recomendado por Noguera et al. (2003), es decir entre 0.75 y $3.49 \mathrm{dS} / \mathrm{m}$ y dado que la CE expresa de manera aproximada la concentración de sales ionizadas en la solución del sustrato (Burés 1997), la baja CE podría compensarse mediante el suficiente suministro de nutrientes a través de la fertirrigación. Por su parte, Díaz et al. (2007) consignan que los compost no deben exceder $2.0 \mathrm{dS} / \mathrm{m}$ para cultivos en vivero. En vista de lo anterior, la CE de las mezclas con compost sí fueron satisfactorias, ya que se ubicaron dentro de este rango ( 0.8 a $1.5 \mathrm{dS} / \mathrm{m})$, así como el compost al $100 \%(0.43 \mathrm{dS} / \mathrm{m})$. A este respecto, Rodríguez et al. (2010) reportaron un valor de CE para compost de bagazo de agave de $1.59 \mathrm{dS} / \mathrm{m}$.

Relación carbono/nitrógeno $(\mathbf{C} / \mathbf{N})$. Tanto el polvo de coco como la turba presentaron relaciones $\mathrm{C} / \mathrm{N}$ altas ya que son materiales muy estables y con gran contenido de fibra. Abad et al. (2002) reportan una relación $\mathrm{C} / \mathrm{N}$ entre 75 y 186 para el polvo de coco originario de seis países productores; rango dentro del cual se encuentra el material aquí evaluado (111.47). Por otra parte, Estévez et al. (2009) reportan una relación $\mathrm{C} / \mathrm{N}$ de 72 para la turba, que fue similar a la del presente estudio (71.16). Noguera et al. (2003) recomiendan una relación $\mathrm{C} / \mathrm{N}$ entre 20 y 40, como indicador de madurez y estabilidad. En este sentido, las mezclas de compost cumplieron esta condición (19.9 a 30.7), a diferencia del compost al $100 \%$ que alcanzó un valor por debajo del mínimo (16.3). No obstante, este valor coincide con lo expresado por Íñiguez et al. (2005), quienes reportaron una relación $\mathrm{C} / \mathrm{N}$ de 17.8 , sin embargo, por debajo de lo que informan Rodríguez et al. (2010), quienes cuantificaron un valor de 24.0 para compost de bagazo de agave tequilero. La relación $\mathrm{C} / \mathrm{N}$ es un indicador del grado de degradación biológica de un material orgánico. La relación inicial baja de $\mathrm{C} / \mathrm{N}<$ 30 implica que los materiales pueden sufrir un proceso de transformación biológica (mineralización y humificación). En cambio, si la relación $\mathrm{C} / \mathrm{N}$ es mayor a 40, la actividad biológica disminuye hasta llegar a un nivel con muy poca degradación $(\mathrm{C} / \mathrm{N}>$ 85) (Bueno et al. 2008). Por otra parte, la relación $\mathrm{C} / \mathrm{N}$ ideal para un compost madura es cercana a 10 , similar al humus, sin embargo, en la práctica se suele considerar que un compost está suficientemente estable o maduro cuando la $\mathrm{C} / \mathrm{N}$ es $<20$ (Bueno et al. 2008); esta condición se cumplió en el compost al $100 \%$ (16.3). Las diferencias estadísticas entre los tratamientos fueron altamente significativas $(\mathrm{p}<0.001)$, en donde únicamente las mezclas de $70 \%$ y $100 \%$ resultaron estadísticamente iguales.

Nitrógeno total Kjeldahl (NTK). Los sustratos comerciales arrojaron concentraciones más bajas de $\mathrm{N}$ que los sustratos con compost, y en éstos el $\mathrm{N}$ aumentó conforme se incrementó la proporción de compost en el sustrato, al grado que el compost al $100 \%$ fue el material con la mayor concentración, ya que alcanzó $1.38 \%$ de N. Este valor se aproxima a lo reportado por Íñiguez et al. (2005) y Rodríguez et al. (2010) quienes señalan valores de $1.0 \%$ y $0.83 \%$ de $\mathrm{N}$ respectivamente, en compost de bagazo de agave tequilero. La turba y los tratamientos $30 \%$ y $50 \%$ $(\mathrm{v} / \mathrm{v})$ fueron estadísticamente iguales $(\mathrm{p}<0.001)$.

Fósforo. Según se aprecia en el cuadro II, todos los materiales evaluados en el estudio mostraron una concentración de fósforo mayor a los parámetros recomendados (0.0006- $0.001 \% \mathrm{P})$. Todas las mezclas con compost fueron estadísticamente diferentes al polvo de coco y la turba $(\mathrm{p}<0.001)$. En este sentido, el menor valor correspondió a la turba con $0.03 \%$ y el mayor al compost al $100 \%$ con $0.28 \%$ de fósforo. Este último valor se acercó al reportado por Íñiguez et al. (2005) para compost de bagazo de agave, el cual fue de $0.1 \%$. Por el contrario, fue muy superior a lo reportado por Rodríguez et al. (2010) con 0.005 $\%$. Pinamonti y Sicher (2005) establecen que las concentraciones de fósforo para compost en general se ubican entre 0.25 y $0.74 \%$, intervalo en el cual se ubica el compost al $100 \%$ del presente estudio. El fósforo del compost proviene de algunas enzimas y proteínas, ATP, RNA, DNA y fitina del residuo vegetal y su concentración en las plantas alcanza hasta el 1.0\% del peso seco (Jones 2012).

Potasio. El polvo de coco presentó la concentración de K más alta (1.63 \%), lo cual es una característica típica de este material (Abad et al. 2002). Burés (1997) señala que el contacto con el agua de mar en las zonas de origen o a veces la elevada salinidad 
del agua de lavado utilizada para la producción del polvo de coco puede generar problemas en la $\mathrm{CE}$, y por lo tanto, incrementar el contenido de potasio, sodio o cloro. Por otra parte, a la turba correspondió el valor más bajo $(0.07 \%)$ y las mezclas de compost variaron entre $0.32 \%$ y $0.37 \%$. El compost al 100 $\%$ registró un contenido de $0.38 \%$ de $\mathrm{K}$ que excede ampliamente la recomendación y fue mayor a lo reportado por Íñiguez et al. (2005) con $0.20 \%$ para compost de bagazo de agave tequilero. Pinamonti y Sicher (2005) manifiestan que un contenido entre 0.37 y $0.54 \%$ de $\mathrm{K}$ es común en los compost en general, rango dentro del cual se encuentra el compost $100 \%$ del presente estudio (0.38\%). Las diferencias estadísticas entre los tratamientos fueron altamente significativas $(\mathrm{p}<0.001)$.

Calcio. De acuerdo con el cuadro II, todos los materiales evaluados cubrieron la recomendación de Ca para sustratos (>0.02\%). Las tres mezclas con compost registraron altas concentraciones de $\mathrm{Ca}(3.60-4.87 \%)$ y fueron estadísticamente iguales $(p<0.001)$, en tanto que el compost al $100 \%$ mostró el valor más alto $(5.70 \%)$. Estos resultados se debieron a la formación de carbonatos de calcio y magnesio como consecuencia de la mineralización que ocurre durante el proceso de compostaje (Gutser y Ebertseder 2002).

Magnesio. Igualmente, en todos los casos se obtuvieron resultados compatibles con los valores recomendados en la concentración óptima de $\mathrm{Mg}$ $(>0.007 \%)$. Las mezclas con compost registraron valores que coinciden también con lo reportado por Gutser y Ebertseder (2002) para compost en general. El Mg de los compost proviene de la clorofila y algunas enzimas contenidas en el material vegetal; el contenido en las plantas varía entre 0.15 y $1.5 \%$ del peso seco (Jones 2012).

\section{Propiedades físicas de los sustratos}

En el cuadro IV se presentan las características físicas de los sutratos evaluados junto con los resultados de la prueba de Tukey. En tanto, el cuadro V muestra el resumen del análisis de varianza obtenido de dichas características.

CUADRO V. RESUMEN DEL ANÁLISIS DE VARIANZA DE LOS ANÁLISIS FÍSICOS DE LOS SUSTRATOS

\begin{tabular}{lccc}
\hline Análisis & $\begin{array}{c}\text { Error } \\
\text { experimental } \\
(\mathrm{s})\end{array}$ & $\begin{array}{c}\text { Coeficiente de } \\
\text { determinación } \\
\text { ajustado }\left(\mathrm{R}^{2}\right)(\%)\end{array}$ & $\begin{array}{c}\text { Nivel de } \\
\text { significancia } \\
(\mathrm{p})\end{array}$ \\
\hline $\mathrm{Da}$ & 0.0497 & 95.31 & 0.000 \\
$\mathrm{Dr}$ & 0.0460 & 95.62 & 0.000 \\
EPT & 1.3110 & 99.35 & 0.000 \\
AFD & 0.8702 & 98.32 & 0.000 \\
AR & 0.6733 & 93.62 & 0.000 \\
ATD & 1.0690 & 98.22 & 0.000 \\
\hline
\end{tabular}

$\mathrm{Da}=$ densidad aparente, $\mathrm{Dr}=$ densidad real, $\mathrm{EPT}=$ espacio poroso total, $\mathrm{AFD}=$ agua fácilmente disponible, $\mathrm{AR}=$ agua de reserva, $\mathrm{ATD}=$ agua total disponible

Densidad aparente (Da). La Da en seco de los sustratos evaluados se muestra en cuadro IV, en donde se aprecia que el polvo de coco alcanzó 0.07 $\mathrm{g} / \mathrm{cm}^{3}$ y fue similar a lo reportado por Vargas et al. (2008) $\left(0.08-0.18 \mathrm{~g} / \mathrm{cm}^{3}\right)$, mientras que la turba mostró un valor de densidad de $0.11 \mathrm{~g} / \mathrm{cm}^{3}$, parecido a lo reportado por Abad et al. (1995). Ambos

CUADRO IV. CARACTERÍSTICAS FÍSICAS DE LOS SUSTRATOS COMERCIALES Y MEZCLAS A BASE DE COMPOST

\begin{tabular}{lcccccc}
\hline \multicolumn{1}{c}{ Sustratos } & $\begin{array}{c}\mathrm{Da} \\
\mathrm{g} / \mathrm{cm}^{3}\end{array}$ & $\begin{array}{c}\mathrm{Dr} \\
\mathrm{g} / \mathrm{cm}^{3}\end{array}$ & $\begin{array}{c}\text { EPT } \\
\%\end{array}$ & $\begin{array}{c}\text { AFD } \\
\%\end{array}$ & $\begin{array}{c}\text { AR } \\
\%\end{array}$ & $\begin{array}{c}\text { ATD } \\
\%\end{array}$ \\
\hline Polvo de coco & $0.07^{\mathrm{d}}$ & $1.56^{\mathrm{c}}$ & $95.8^{\mathrm{a}}$ & $22.7^{\mathrm{b}}$ & $11.1^{\mathrm{b}}$ & $33.8^{\mathrm{b}}$ \\
Turba & $0.11^{\mathrm{d}}$ & $1.54^{\mathrm{c}}$ & $92.7^{\mathrm{a}}$ & $32.6^{\mathrm{a}}$ & $11.6^{\mathrm{b}}$ & $44.2^{\mathrm{a}}$ \\
Compost $30 \%$ & $0.30^{\mathrm{c}}$ & $1.94^{\mathrm{b}}$ & $67.0^{\mathrm{c}}$ & $12.7^{\mathrm{d}}$ & $16.5^{\mathrm{a}}$ & $46.9^{\mathrm{a}}$ \\
Compost $50 \%$ & $0.40^{\mathrm{c}}$ & $1.97^{\mathrm{ab}}$ & $76.0^{\mathrm{b}}$ & $14.6^{\mathrm{d}}$ & $12.8^{\mathrm{b}}$ & $27.3^{\mathrm{d}}$ \\
Compost $70 \%$ & $0.53^{\mathrm{b}}$ & $2.06^{\mathrm{a}}$ & $60.0^{\mathrm{d}}$ & $19.0^{\mathrm{c}}$ & $9.0^{\mathrm{c}}$ & $28.0^{\mathrm{cd}}$ \\
Compost $100 \%$ & $0.70^{\mathrm{a}}$ & $1.97^{\mathrm{ab}}$ & $53.0^{\mathrm{e}}$ & $19.6^{\mathrm{c}}$ & $9.1^{\mathrm{c}}$ & $30.4^{\mathrm{c}}$ \\
Recomendados $\dagger$ & $<0.40$ & $1.45-2.65$ & $70-85$ & $20-30$ & $4-10$ & $24-40$ \\
\hline
\end{tabular}

$\mathrm{Da}=$ densidad aparente, $\mathrm{Dr}=$ densidad real, $\mathrm{EPT}=$ espacio poroso total, $\mathrm{AFD}=$ agua fácilmente disponible, $\mathrm{AR}=$ agua de reserva, ATD = agua total disponible. $\dagger$ De Boodt y Verdock (1974), Ansorena (1994), Handreck y Black (1994) y Abad (1995). Medias con la misma letra en cada columna son estadísticamente iguales $(\mathrm{p} \leq 0.001)$ 
materiales se ubicaron dentro del valor recomendado para sustratos $\left(<0.4 \mathrm{~g} / \mathrm{cm}^{3}\right)$. Sin embargo, en el caso de las mezclas con compost esta condición sólo se cumplió para los tratamientos $30 \%$ y $50 \%$, con 0.30 y $0.40 \mathrm{~g} / \mathrm{cm}^{3}$ respectivamente. En cambio, la mezcla con compost al $100 \%$ registró el valor mayor $\left(0.70 \mathrm{~g} / \mathrm{cm}^{3}\right)$ debido que contenía grava y suelo incorporados por la maquinaria en el sitio de compostaje. Este resultado difiere de otros compost de bagazo de agave, como los reportado por Íñiguez y Crohn (2004), con $0.35 \mathrm{~g} / \mathrm{cm}^{3}$, y Rodríguez et al. (2010), con $0.173 \mathrm{~g} / \mathrm{cm}^{3}$, pero hay que considerar que el compostaje que aplicaron estos autores se realizó sobre piso firme, sin intrusiones de suelo y grava. $\mathrm{La} \mathrm{Da}$ es importante para las plantas que requieren riegos frecuentes, oxigenación y anclaje suficiente para evitar problemas debidos a la acción del viento; bajo estas circunstancias, la Da puede ser tan baja como $0.15 \mathrm{~g} / \mathrm{cm}^{3}$ (Urrestarazu 2004, Raviv y Lieth 2008). Por el contrario, los cultivos en contenedor expuestos al exterior requieren una mayor $\mathrm{Da}$ a fin de evitar la inestabilidad ocasionada por el viento (Raviv y Lieth 2008). Entre los tratamientos se registraron diferencias estadísticas altamente significativas $(\mathrm{p}<$ 0.001) debido al material orgánico utilizado (Pire y Pereira 2003).

Densidad real (Dr). Para Noguera et al. (2003) el intervalo óptimo de Dr para sustratos es de 1.45 a $2.65 \mathrm{~g} / \mathrm{cm}^{3}$. De acuerdo con el cuadro IV, tanto el polvo de coco, como la turba se encontraron dentro de este rango (1.56 y $1.54 \mathrm{~g} / \mathrm{cm}^{3}$, respectivamente). De la misma forma, todos los sustratos con compost $\left(1.94 \mathrm{a} 2.06 \mathrm{~g} / \mathrm{cm}^{3}\right)$ se ubicaron dentro de los valores óptimos, así como el compost al $100 \%\left(1.97 \mathrm{~g} / \mathrm{cm}^{3}\right)$. Rodríguez et al. (2010) señalan como apropiado un valor de $1.38 \mathrm{~g} / \mathrm{cm}^{3}$ para compost de bagazo de agave tequilero. Los resultados obtenido de los sustratos con compost fueron casi todos estadísticamente similares $(\mathrm{p}<0.001)$, pero diferentes a los testigos.

Espacio poroso total (EPT). Varios de los materiales evaluados mostraron un EPT dentro del intervalo recomendado para sustratos: $70-85 \%$. En este rango se encuentra el balance ideal entre el aire, necesario para la respiración de las raíces de las plantas, y el agua que se encuentra en los microporos (Burés 1997). Las mezclas con compost al $70 \%$ y $100 \%$ tuvieron los valores más bajos (intervalo: 60.0 a $53.0 \%$ ). Esto se explica por la mayor densidad del compost como resultado del intenso proceso de degradación a través del cual quedaron menos espacios porosos, y por consiguiente, se redujo la granulometría (Rodríguez et al. 2010). El resultado del EPT del compost al $100 \%$ (53.0 \%) fue menor a lo que reportan Rodríguez et al. (2010) con $76.7 \%$ para bagazo de agave tequilero compostado. Los sustratos comerciales fueron estadísticamente iguales entre sí $(\mathrm{p}<0.001)$, en tanto que todos los sustratos con compost fueron diferentes, siendo el tratamiento de compost al $50 \%(\mathrm{EPT}=76.0 \%)$ y el compost al $30 \%(\mathrm{EPT}=67.0 \%)$ las mezclas con los mejores resultados.

Agua fácilmente disponible (AFD). En el cuadro IV se observa que los sustratos comerciales registraron los resultados más altos de AFD, los cuales fueron estadísticamente diferentes entre sí ( $\mathrm{p}$ $<0.001$ ). Solamente el polvo de coco se ubicó dentro del intervalo recomendado por De Boodt y Verdock (1974) para sustratos: $20-30 \%$, mientras que la turba y el compost al $100 \%$ se encontraron cercanos al rango: $32.6 \%$ y $19.6 \%$, respectivamente. Por otra parte, entre las mezclas el tratamiento compost $70 \%$ fue el que mejor valor registró $(19.0 \%)$ y sólo fue estadísticamente similar al compost al $100 \%$ $(\mathrm{p}<0.001)$. Rodríguez et al. (2010) reportaron una AFD de $21.7 \%$ para compost de bagazo de agave, que se encuentra dentro del rango ideal (20 - 30\%). El AFD representa el porcentaje del volumen de agua que se libera entre 10 a $50 \mathrm{~cm}$ de tensión de una columna de agua sobre el sustrato. Los bajos valores obtenidos en las mezclas con compost pueden deberse al efecto de segregación, el cual es un problema común de separación que ocurre con las partículas menos densas y grandes que se mueven hacia arriba del material durante el transporte (Jones 2005). Cabe destacar que conforme se incrementa la proporción de compost, se incrementa también la $\mathrm{Da}$ $(\mathrm{r}=0.999 ; \mathrm{p}<0.001)$.

Agua de reserva (AR). Como se observa en el cuadro IV, el AR del polvo de coco (11.1\%), la turba (11.6\%) y los compost al 30 y $50 \%$ (16.5 y $12.8 \%$ respectivamente), se encontraron por arriba del intervalo recomendado por Abad (1995), es decir, entre el 4 y $10 \%$. El compost al $50 \%$ fue estadísticamente similar al polvo de coco y a la turba $(\mathrm{p}<0.001)$, en tanto que el compost al $30 \%$ fue diferente al resto de los tratamientos. Solamente el AR de los compost al 70 y $100 \%$ (9.0 y $9.1 \%$ respectivamente), coinciden con el valor recomendado para sustratos (4 - 10\%) y los resultados también son similares a lo reportado por Rodríguez et al. (2010), quienes determinaron un valor de $9.3 \%$ para compost de bagazo de agave tequilero. Estos autores refieren que el bagazo contiene fibras que presentan aberturas y poros que ayudan a retener mayor volumen de agua, y por lo tanto, les permite a las plantas disponer de agua por más tiempo. Otro factor que pudo influir en el resultado fue la 
existencia de una mayor cantidad de partículas finas producidas por el compostaje intenso del bagazo; estas partículas finas son las responsables de retener más agua en los sustratos (Ansorena 1994).

Agua total disponible (ATD). El mayor volumen de ATD lo presentaron la turba, con $44.2 \%$, y el compost al $30 \%(46.9 \%)$, cuyos valores excedieron el rango recomendado por Abad (1995) (24 a $40 \%$ ). Ambos sustratos fueron estadísticamente semejantes $(\mathrm{p}<0.001)$. El polvo de coco y los compost al 50, $70 \mathrm{y}$ $100 \%$ sí se ubicaron dentro de los valores sugeridos, sin embargo, el polvo de coco fue estadísticamente diferente al resto de los tratamientos $(p<0.001)$. Rodríguez et al. (2010) sugieren que el valor de ATD de los compost de bagazo de agave denotan ausencia de parénquima debido al proceso de degradación, lo cual induce una menor capacidad de imbibición con respecto a los sustratos comerciales, hecho que se refleja en la menor capacidad de almacenamiento de agua que éstos.

Distribución del tamaño de partículas (DTP). Este factor afecta la porosidad de un sustrato, es decir, la capacidad que éste tiene de resistir un mal drenaje y bajos niveles de oxígeno. Ansorena (1994) señala que en las dimensiones de partículas comprendidas entre 1 y $10 \mathrm{~mm}$, tanto la porosidad como la cantidad de agua retenida varían poco con el tamaño de las partículas. En la distribución de partículas de los sustratos evaluados, fue clara la tendencia de las mezclas de compost y compost al $100 \%$ de presentar un contenido similar de partículas $>1 \mathrm{~mm}$ (promedio $45.1 \%$ ), que en general fue $20.8 \%$ mayor al promedio de los sustratos comerciales, los que a su vez registraron también un contenido similar entre sí (promedio $24.3 \%$ ). La tendencia se invirtió con la fracción $<1 \mathrm{~mm}$, en donde los sustratos comerciales mostraron en promedio $20.8 \%$ mayor cantidad de partículas finas que los sustratos con compost. Las diferentes magnitudes y tendencias de la distribución de partículas pueden apreciarse en la figura 1. Cadahía (2005) señala que en los materiales de textura gruesa $(>0.9 \mathrm{~mm})$ predominan poros grandes que retienen cantidades reducidas de agua y están bien aireados. En otras palabras, los sustratos a base de compost, comparados con los sustratos comerciales, registraron una mejor condición para la circulación de aire.

Por otra parte, Ansorena (1994), refiere que al pasar a partículas de tamaño $<1 \mathrm{~mm}$, se observa un brusco descenso de la porosidad y el aumento de la retención de agua. A este respecto, Handreck (1983), reporta que la fracción $<0.5 \mathrm{~mm}$ presenta la máxima influencia en el espacio poroso drenable y en la

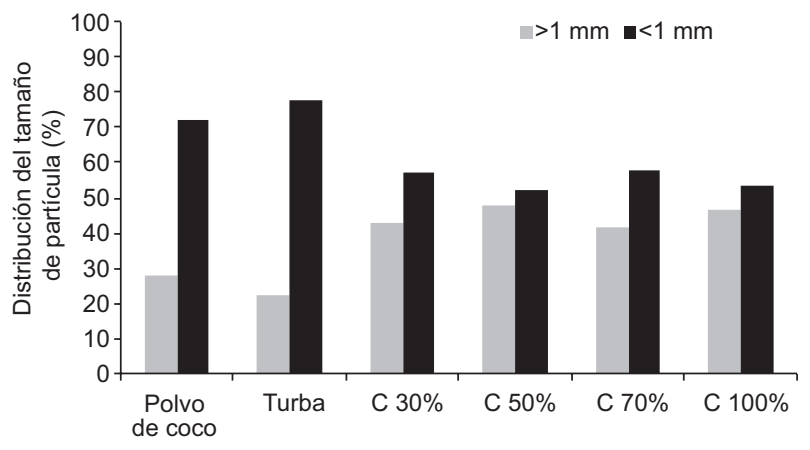

Fig. 1. Distribución del tamaño de partículas de los sustratos comerciales y del compost en porcentaje. $\mathrm{C}=$ compost

retención de agua en mezclas basadas en corteza de pino, es decir, los materiales finos retienen grandes cantidades de agua difícilmente disponible y están mal aireados (Cadahía 2005).

Debido al hecho de que los sustratos con compost contienen un apreciable porcentaje de partículas finas, menores a $1.0 \mathrm{~mm}$, resultan recomendables para emplearse en el cultivo de plantas de ciclo corto, ya que una mezcla con abundancia de partículas finas es mejor para la germinación de semillas (Jones 2005). En este caso, las partículas mayores a $2.38 \mathrm{~mm}$ deberán de ser eliminadas para mejorar los condiciones de humedad del sustrato (Jones 2005). Sin embargo, si se requiere aplicar esos sustratos para los cultivos de ciclo largo en contenedor, se tendrían que desechar las partículas menores a $0.59 \mathrm{~mm}$ cuando sobrepasen del 20 al $30 \%$ y conservar las partículas mayores a $2.38 \mathrm{~mm}$, para evitar deficiencias del drenaje, ya que las mezclas gruesas son mejores para plantas de flor y las leñosas ornamentales (Jones 2005).

\section{CONCLUSIONES}

En este trabajo se pudo demostrar que las formulaciones de compost de bagazo de agave con polvo de coco podrían reemplazar el uso de turba y polvo de coco, los cuales son materiales comerciales que sobresalen por su alto precio. Las formulaciones elaboradas a base de compost tienen características químicas y físicas satisfactorias para ser utilizadas como sustratos para plantas cultivadas en contenedor. La formulación con $30 \%$ de compost fue la mezcla con la mayor cantidad de parámetros químicos cuyos resultados se acercaron más a seis estándares recomendados para sustratos, tales como: $\mathrm{pH}, \mathrm{CIC}$, $\mathrm{CE}, \mathrm{C} / \mathrm{N}, \mathrm{Ca}$ y $\mathrm{Mg}$, de hecho esta mezcla superó a los sustratos comerciales. Le siguieron los compost 
al $50 \%$ y $70 \%$, y por último el compost al $100 \%$. En todos los sustratos con compost fue mayor el contenido de $\mathrm{N}, \mathrm{P}$ y Ca, respecto al polvo de coco y la turba, y únicamente el polvo de coco mostró valores mayores de K. Las diferencias en el contenido nutrimental de los sustratos son debidas a la naturaleza propia de los materiales, y en el caso de los mezclas con compost, el mayor contenido nutrimental podría permitir la reducción de la fertilización tradicional sin afectar significativamente el rendimiento de los cultivos agrícolas. Esto puede considerarse como una mejora en lo que se refiere a la fertilización de sustratos en los sistemas de producción bajo invernadero. En cuanto a las propiedades físicas, las mezclas con $50 \%$ y $100 \%$ de compost fueron los tratamientos que registraron los mejores resultados en cuatro de los seis estándares recomendados. Le siguieron los compost al $30 \%$ y $70 \%$. En el agua de reserva (AR) el compost al $50 \%$ fue estadísticamente igual $(\mathrm{p}<$ 0.001 ) al polvo de coco y a la turba, mientras que el compost al $30 \%$ fue similar a la turba en agua total disponible (ATD).

El aprovechamiento de los desechos compostados podría traer consigo otros beneficios, tales como la reducción del impacto de la contaminación ambiental a través de la producción local de sustratos destinados al sector de los cultivos protegidos, el cual tiene una demanda cada vez más creciente de estos materiales.

\section{REFERENCIAS}

Abad M. (1995). Sustratos para el cultivo sin suelo. En: El cultivo del tomate. (F. Nuez, Ed.). Ediciones MundiPrensa, Madrid, España, pp. 131-265.

Abad M., Noguera P. y Noguera V. (1995). Turbas para semilleros. Memorias. II Jornadas sobre semillas y semilleros hortícolas. Jornadas 35/96. Junta de Andalucía. Consejería de Agricultura y Pesca. Dirección General de Investigación Agraria. Sevilla, España. 29 al 31 de mayo, 1995.

Abad M., Noguera P., Puchades R., Maquieira A. y Noguera V. (2002). Physico-chemical and chemical properties of some coconut coir dusts for use as peat substitute for containerized ornamental plants. Biores. Technol. 82, 241-245.

DOI: 10.1016/S0960-8524(01)00189-4

Alexander R. (2001). Compost utilization in landscapes. En: Compost utilization in horticultural cropping systems (P.J. Stoffela y B.A. Kahn, Eds.). Lewis Publishers, Boca Raton, Florida, USA, pp. 151-175.

DOI: 10.1201/9781420026221.ch7
Ansorena J. (1994). Sustratos. Propiedades y caracterización. Mundi Prensa. Madrid, España, 172 pp.

Bucher A. (2008). Recyclingstoffe im Gartenbau (CDROM). Institut für Gartenbau, Fachhochschule Weihenstephan, Freising, Alemania.

Bueno P., Díaz M.J. y Cabrera F. (2008). Factores que afectan el proceso de compostaje. En: Compostaje. (J. Moreno y R. Moral, Eds.). Ediciones Mundi-Prensa, Madrid, España, pp. 95-109.

Burés S. (1997). Sustratos. Ediciones Agrotécnicas, S.L. Madrid, España, 341 pp.

Cadahía C. (Ed.) (2005). Fertirrigación. Cultivos hortícolas, frutales y ornamentales. 3a. ed. Ediciones MundiPrensa, Madrid, España, 681 pp.

CNIT (2013). El tequila/agave. Cámara Nacional de la Industria del Tequila. [en línea]. http://www.tequileros. org 06/10/2013.

Crespo M., González D.R., Rodríguez R., Rendón L.A., del Real J.I. y Torres J.P. (2013). Evaluación de composta de bagazo de agave como componente de sustratos para producir plántulas de agave azul tequilero. Rev. Mex. de Cien. Agríc. 4, 1161-1173.

CRT (2016). Consejo Regulador del Tequila [en línea]. http://www.crt.org.mx/EstadisticasCRTweb/ 17/01/2016.

Day M. y Shaw K. (2001). Biological, chemical, and physical process of composting. En: Compost utilization in horticultural cropping systems. (P.J. Stoffela y B.A. Kahn, Eds.). Lewis Publishers, Boca Raton, Florida, EUA, pp. 17-50. DOI: 10.1201/9781420026221.ch2

De Boodt M. y Verdonck O. (1974). Methods for measuring the water release curve of organic substrates. Acta Hort. 37, 2054-2062.

DOI: $10.17660 /$ ActaHortic. 1974.37 .20

Diaz L.F., de Bertoldi M., Bibdlingmaier W. y Stentiford E. (2007). Compost science and technology, vol. 8. Elsevier. Amsterdam, Holanda, $364 \mathrm{pp}$.

DOI: $10.1016 / \mathrm{s} 1478-7482(07) \times 8002-3$

EPA(2000). EPA-832-F-00-064. Biosolid technology fact sheet. Land application of biosolids. Environmental Protection Agency, EUA, Washington D.C. [en línea]. https://www.epa.gov/biosolids/land-applicationbiosolids 25/02/2011.

Estévez I., Seoane S., Núñez A. y López M.E. (2009). Characterization and evaluation of compost utilized as ornamental plant substrate. Compost Sci. Util. 17 (4), 210-219. DOI: 10.1080/1065657x.2009.10702426

Gouleke C.G. (1977). Biological processing: Composting and hydrolisis. En: Handbook of solid waste management (D.G. Wilson, Ed.). Van Norstrand Reinhold, Nueva York, EUA, pp. 197-225.

Gutser R. y Ebertseder T. (2002). Grundlagen zur Nährstoff-und Sonderwirkung sowie optimalen Einsatz- 
strategien von Komposten im Freiland. En: Handbuch Kompost im Gartenbau. Zentralverband Gartenbau e.V. (P. Menzel y H.J. Brinkjans, Eds.), Bonn, Alemania, pp. 47-72.

Handreck K.A. (1983). Particle size and the physical properties of growing media for containers. Commun. Soil Sci. Plant Anal. 14 (3), 209-222.

DOI: $10.1080 / 00103628309367357$

Harada Y.A., Inoko A., Tadaki M. e Izawa T. (1981). Maturing process of city refuse compost during piling. En: The science of composting. (E. Epstein, Ed.). CRC Press, Boca Raton, Florida, EUA, pp. 107-136. DOI: $10.1080 / 00380768.1981 .10431290$

He X.T., Logan T. J. y Traina S. J. (1995). Physical and chemical characteristics of selected U.S. municipal solid waste compost. J. Environ. Qual. 24 (3), 543552. DOI: $10.2134 /$ jeq1995.00472425002400030022x

Íñiguez G. y Crohn D. M. (2004). Utilization of by-products from the tequila industry. Part 6 . Fertilization of potted geranium with a slaughterhouse waste compost. Rev. Int. Contam. Ambie. 20 (2), 53-58.

Íñiguez G., Acosta N., Martínez L., Parra J. y González O. (2005). Utilización de subproductos de la industria tequilera. Parte 7. Compostaje de bagazo de agave y vinazas tequileras. Rev. Int. Contam. Ambie. 21 (1), 37-50.

Jones J. B. (2005). Hydroponics: A practical guide for the soilless grower. 2a. ed., CRC Press, Boca Raton, Florida, EUA, 423 pp. DOI: 10.1201/9780849331671

Jones J. B. (2012). Plant nutrition and soil fertility manual. 2a. ed. CRC Press. Taylor and Francis Group. Boca Raton, Florida, EUA, 282 pp. DOI: 10.1201/b11577

Maher M., Prasad M. y Raviv M. (2008). Organic soilless media components. En: Soilless culture (M. Raviv and J.H. Lieth, Eds.). Elsevier, Amsterdam, Holanda, pp. 459-504. DOI: 10.1016/b978-044452975-6.50013-7

Martínez F.X. (1993). Propuesta de metodología para la determinación de las propiedades físicas de los sustratos. Acta Hort. 11, 55-66.

Minitab 17 (2015). Languaje Pack-español. Minitab® Inc. v.17.2.1. EUA.

Noguera P., Abad M., Puchades R., Maquieira A. y Noguera V. (2003). Influence of particle size on physical and chemical properties of coconut coir dust as container medium. Commun. Soil Sci. Plant Anal. 34 (3-4), 593-605. DOI: 10.1081/CSS-120017842
Pinamonti F. y Sicher L. (2005). Compost utilization in fruit production systems. En: Compost utilization in horticultural cropping systems. (P. J. Stoffela y B. A. Kahn, Eds.). Lewis Publishers, Boca Raton, Florida, EUA, pp. 177-200.

DOI: $10.1201 / 9781420026221 . c h 8$

Pire R. y Pererira A. (2003). Propiedades físicas de componentes de sustratos de uso común en la horticultura del estado de Lara, Venezuela. Propuesta metodológica. Bioagro 15, 55-63.

Raviv M. y Lieth J.H. (2008). Soilless culture. Theory and practice. Elsevier. EUA, 587 pp. DOI: 10.1016/b978-044452975-6.50003-4

Rodríguez L., Fernández F., Íñiguez G., RodríguezGuzmán, E., Rodríguez-Díaz E. y Arriaga M. (2010). Utilización de bagazo de agave como sustrato para producción de plántulas de tomate. Scientia-CUCBA 12 (1-2), 11-15.

Rodríguez R., Alcántar E.G., Íñiguez G., Zamora F., García P.M., Ruiz M. y Salcedo E. (2010). Caracterización física y química de sustratos agrícolas a partir de bagazo de agave tequilero. Interciencia 35 (7), 515-520.

Rodríguez R., Jiménez F., del Real J.I., Salcedo E., Zamora F. e Íñiguez G. (2013). Utilización de subproductos de la industria tequilera. Parte 11. Compostaje de bagazo de agave crudo y biosólidos provenientes de una planta de tratamiento de vinazas tequileras. Rev. Int. Contam. Ambie. 29 (4), 303-313.

Soliva M. (1998). Aplicación del compostaje a la obtención de productos alternativos a los sustratos tradicionales. En: Tecnología de sustratos: aplicación a la producción viverística ornamental, hortícola y forestal (S. Pastor y J. Narciso, Eds.). Universidad de Lleida, Lérida, España, pp. 51-93.

TMECC (2001). Test method for the examination of composting and compost. US Composting Council Research and Education Foundation and the U.S.D.A. CD- ROM companion [CD-ROM computer file] U.S.A.

Urrestarazu M. (2004). Tratado de cultivo sin suelo. $3 \mathrm{a}$. ed. Ediciones Mundi-Prensa, Madrid, España, 914 pp.

Vargas P., Castellanos J.Z., Sánchez P., Tijerina L., López R.M. y Ojodeagua J.L. (2008). Caracterización física, química y biológica de sustratos de polvo de coco. Rev. Fitotec. Mex. 31 (4), 375-381. 